\title{
Desarrollo de una aplicación en el robot NAO H25 para la enseñanza y comprobación de la operación multiplicación con el método Maya
}

\section{Development of an application on the NAO H25 robot for the teaching and checking of the multiplication operation with Mayan method}

\author{
GUTIERREZ-LUGO, Marco Antonio †*, MENDOZA-PÉREZ, Marco Alberto y CRUZ-FLORES, René \\ Guadalupe
}

Centro Universitario UAEM Valle de Chalco.

ID $1^{\text {er }}$ Autor: Marco Antonio, Gutierrez-Lugo / ORC ID -0000-0002-5520-2983, CVU CONACYT ID: 970762

ID $1^{\text {er }}$ Coautor: Marco Alberto, Mendoza-Pérez / ORC ID -0000-0003-4911-4757, CVU CONACYT ID: 1000527

ID $2^{\mathrm{do}}$ Coautor: René Guadalupe, Cruz-Flores / ORC ID -0000-0002-261814245, CVU CONACYT ID: 450072

DOI: $10.35429 /$ JEA.2019.18.6.19.28

Recibido: 19 de Enero, 2019; Aceptado 02 de Marzo, 2019

\begin{abstract}
Resumen
En el presente documento de investigación se muestra el desarrollo de una aplicación implementada en el robot humanoide NAO H25, utilizando la metodología de Análisis, Diseño, Desarrollo, Implementación y Evaluación (ADDIE). Actualmente en la mayoría de las instituciones públicas de educación básica, no se cuenta con tecnología robótica, que el docente utilice para la enseñanza de un tema en particular. La finalidad de la aplicación es que funcione como un recurso didáctico, con el cual profesores y alumnos de educación básica puedan recurrir como forma de apoyo para la enseñanza de la operación multiplicación, dicha aplicación es capaz de hacer que el robot NAO H25 explique la operación multiplicación de forma aritmética y que realice su comprobación por medio del método maya. Cuando la aplicación se implementa en el robot NAO H25, este funge como capacitador en el tema de multiplicaciones, siguiendo una secuencia didáctica como forma de apoyo para el desarrollo de la explicación. Por el momento se realizaron pruebas en un grupo de 32 estudiantes de la carrera de Ingeniería en Computación de este centro universitario. Donde se encontró que el $94 \%$ se le hizo interesante que el robot fungiera como capacitor. El $97 \%$ le gustaría que se realizaran más aplicaciones de este tipo para el aprendizaje de otros temas. El desarrollo de la aplicación es con el motivo de obtener un aprendizaje significativo en los alumnos de tercer año de educación básica, fomentando el interés por la tecnología.
\end{abstract}

Aplicación, Aprendizaje significativo, Método maya, Robot NAO H25

\begin{abstract}
This research document shows the development of an application implemented in the humanoid robot NAO H25, using the methodology of Analysis, Design, Development, Implementation and Evaluation (ADDIE). Currently, in most public institutions of basic education, there is no robotic technology that the teacher uses for teaching a particular subject. The purpose of the application is that it works as a didactic resource, with which teachers and students of basic education can use as a form of support for the teaching of the multiplication operation. This application is capable of making the NAO $\mathrm{H} 25$ robot explain the multiplication operation in an arithmetic way and carry out its verification by means of the Mayan method. When the application is implemented in the NAO H25 robot, it acts as a trainer in the topic of multiplications, following a didactic sequence as a form of support for the development of the explanation. For the moment, tests were carried out on a group of 32 students of the computer engineering degree at this university centre. Where it was found that $94 \%$ were interested in the robot acting as a capacitor. $97 \%$ would like more applications of this type to be made for learning other subjects. The development of the application is with the motive of obtaining a significant learning in the students of third year of basic education, fomenting the interest by the technology.
\end{abstract}

Application, Mayan Method, Robot NAO H25, Significant learning

Citación: GUTIERREZ-LUGO, Marco Antonio, MENDOZA-PÉREZ, Marco Alberto y CRUZ-FLORES, René Guadalupe. Desarrollo de una aplicación en el robot NAO H25 para la enseñanza y comprobación de la operación multiplicación con el método Maya. Revista de Aplicaciones de la Ingeniería. 2019. 6-18: 19-28

\footnotetext{
* Correspondencia del Autor (Correo electrónico: antoniolugo23279@gmail.com)

$\dagger$ Investigador contribuyendo como primer autor.
} 


\section{Introducción}

La robótica es una ciencia perteneciente a la tecnología, donde se estudia y desarrollan máquinas capaces de desempeñar una tarea por sí misma. Algunas de las ciencias que son utilizadas en la robótica son: Informática, Electrónica, Mecánica, Álgebra, Autómatas programables, Máquinas de estados, entre otras ciencias más.

Con la robótica podemos realizar trabajos o acciones de una manera más sencilla, de igual forma ayuda a la educación por medio de la robótica educativa. A través de la robótica educativa y el uso de referentes pedagógicos y didácticos, es posible apoyar los procesos de enseñanza y aprendizaje de la comunidad académica, con herramientas tecnológicas (Pinto, Barrera \& Pérez, 2010).

La ciencia de la robótica no solo se usa para beneficios del sector industrial y de los servicios, también se utiliza en las aulas de clase, posibilitando la elaboración de novedosos ambientes para el aprendizaje.

El origen del término robot proviene de la palabra checa "robota" que significa trabajo, en 1921 el dramaturgo checo Karel Capek introdujo el término robot en su obra "R. U. R. Rossum's Universal Robots" en donde nos habla acerca de una fábrica que construye máquinas con apariencia humana para llevar acabo cierto tipo de tareas. Una mejor definición es la que nos proporciona la RIA (Robot Institute of América) la cual establece que un robot es una maquina manipuladora, multifuncional, reprogramable, diseñado para mover material, herramientas o algún otro dispositivo por medio de movimientos programados para la ejecución de un cierto tipo de tareas.

Un robot humanoide o androide es una máquina antropomórfica capaz de imitar las funciones básicas del ser humano tales como caminar, hablar, ver, recolectar, limpiar y trasladar objetos (Reyes, 2011, P. 10).

El robot NAO H25 pertenece a la gama de robots humanoides de la empresa Aldebarán Robotics, su funcionamiento es de manera autónoma o tele-operada desde un puesto de programación.
Cuenta con 25 grados de libertad, permitiendo movimientos, posee módulos de software embebidos que permite el reconocimiento de voz y de texto a voz, de igual manera tiene bocinas y micrófonos, para la detección de formas utiliza 2 cámaras, tiene la habilidad de comunicarse por medio de LED'S. Posee un alto grado de interacción al incluir inteligencia artificial, esto le permite reconocer $\mathrm{y}$ aprender cualquier rostro u objeto, haciendo posible la interacción Humano-Robot.

Omnidireccional es la capacidad con la que cuenta un robot para moverse en cualquier dirección. El software embebido es un pequeño sistema que procesa información en tiempo real, se usa para cubrir una necesidad específica.

La programación para el robot se usa para introducir una serie de instrucciones necesarias en su sistema de control, para que pueda realizar tareas de forma predeterminada, para la programación del robot hay formas distintas de hacerlo, como son: programación guiada o directa y programación textual o indirecta.

Programación guiada o directa: en este tipo de programación el usuario interviene de forma directa, ya que ejecuta manualmente cada uno de los movimientos que realiza el robot, cada movimiento que se realiza se almacena en la memoria del robot, dando la posibilidad de repetirlos posteriormente, es una forma fácil de programar el robot, pero con la inconveniencia de no tener en cuenta las variaciones del entorno.

Programación textual o indirecta: esta forma de programación se realiza con la ayuda de lenguajes de programación como Python, $\mathrm{C}++$, Java, entre otros lenguajes más. El fin de estos lenguajes es formar un conjunto de instrucciones, que se guardara en la memoria del robot, para que posteriormente estas instrucciones sean ejecutadas por el robot. Con esta forma de programación es posible realizar operaciones complejas y se puede tener en cuenta las variables del entorno.

Como definición de aprendizaje podemos mencionar que son procesos por los cuales se pueden modificar habilidades, destrezas, conductas o ideas por medio de resultados de estudios, experiencias, razonamiento u observación, todo cambio es duradero por medio de la práctica del conocimiento.

GUTIERREZ-LUGO, Marco Antonio, MENDOZA-PÉREZ, Marco Alberto y CRUZ-FLORES, René Guadalupe. Desarrollo de una aplicación en el robot NAO H25 para la enseñanza y comprobación de la operación multiplicación con el método Maya. Revista de Aplicaciones de la Ingeniería. 2019. 
La enseñanza es la práctica de actividades que llevan a los alumnos a conocer, aprender e instruir en su aprendizaje de nuevas habilidades (Anónimo, sf).

El aprendizaje significativo es el proceso por el cual una persona recolecta la información, la selecciona, organiza y establece relaciones con el conocimiento que ya se tenía anteriormente. Esta forma de aprendizaje se da cuando el nuevo contenido se relaciona con experiencias vividas $\mathrm{y}$ otros conocimientos adquiridos. Cuando se produce el aprendizaje significativo, los modelos mentales creados a través del tiempo y la experiencia determinan el cómo veremos la información y el cómo la iremos gestionando (Álvarez, 2016).

La estrategia didáctica es el proceso por el cual el docente elige las técnicas y actividades que puede utilizar con el fin de alcanzar los objetivos propuestos, de fomentar el auto aprendizaje interactivo y el aprendizaje colaborativo de los alumnos (Velasco, 2010).

El recurso didáctico es un material de cualquier tipo que es utilizado para el proceso de la enseñanza, este tiene el objetivo de facilitar la enseñanza al profesor y para el alumno tiene la intención de que adquiera nuevo conocimiento de forma duradera.

El desarrollo e implementación de la aplicación en el Robot NAO H25 para la enseñanza y comprobación de la operación multiplicación con el método maya, se realizó con la ayuda de la metodología de Análisis, Diseño, Desarrollo, Implementación y Evaluación (ADDIE). Esta metodología es utilizada como base para el diseño instruccional, consta de 5 etapas, cada fin de una etapa es el inicio de la siguiente. El diseño de este recurso didáctico con esta metodología, tiene como objetivo que los objetos de enseñanza sean claros, de fácil comprensión y generen en los alumnos de educación básica aprendizajes significativos.

Actualmente en la mayoría de las instituciones públicas de educación básica, no se cuenta con tecnología robótica que el docente utilice para la enseñanza de un tema en particular.
Por tal motivo se desarrolló e implemento la aplicación en el Robot NAO H25 para la enseñanza y comprobación de la operación multiplicación con el método maya, se realizó con la ayuda de la metodología de Análisis, Diseño, Desarrollo, Implementación y Evaluación (ADDIE), esta metodología es utilizada como base para el diseño instruccional, consta de 5 etapas, cada fin de etapa es el inicio de la siguiente.

Se realizaron pruebas en un grupo de 32 alumnos de la carrera de Ingeniería en Computación del Centro Universitario UAEM Valle de Chalco. El diseño de este recurso didáctico con ayuda de esta metodología, tiene como objetivo que los objetos de enseñanza sean claros, de fácil comprensión y generen en los alumnos de educación básica aprendizajes significativos.

\section{Metodología}

Como apoyo para el desarrollo de la aplicación se utilizó la metodología ADDIE, en este apartado se describen cada uno de los elementos de dicha metodología:

\section{Análisis}

Uno de los principales problemas que se presentan en la materia de matemáticas (en educación básica, tercer año de primaria), se encuentra cuando los alumnos comienzan a aprender a multiplicar, basándose en el libro de Desafíos Matemáticos (2014) de tercer año de primaria, se encuentran temas (Bloque I, del tema 6 al tema 10) en donde se muestra a los alumnos ejemplos de memorización de productos (multiplicaciones) de algunos números (SEP, 2014).

Tomando en cuenta lo anterior el proceso de enseñanza-aprendizaje de las multiplicaciones es por medio de la memorización de las tablas de multiplicar (las más esenciales son del 1 al 10), con ello se realizan ejercicios para reforzar lo aprendido por los alumnos. El libro de Desafíos Matemáticos incorpora ejemplos y ejercicios que ayudan al alumno en su aprendizaje (SEP, 2014). 
A medida que se avanza en el tema de las multiplicaciones podemos detectar que uno de los problemas más difíciles de enfrentar es cuando la complejidad de la multiplicación va en aumento, presentándose ejercicios o problemas de más dígitos en una o en ambas partes de la multiplicación (multiplicando y multiplicador), con esto es más fácil que los alumnos se confundan y puedan hasta equivocarse.

En ese momento es donde actúa NAO H25 para ayudar a los alumnos a aprender a multiplicar y comprobar sus resultados, esto se realiza por medio de una clase que es impartida por NAO H25. La forma en que se comprobaran los resultados es por medio del método maya, mismo que sirve como un auxiliar a la hora de hacer multiplicaciones.

El método de la multiplicación maya consiste en la colocación de líneas rectas paralelas y perpendiculares, donde cada digito indica el número de rectas que serán representadas, una de las ventajas es que no es necesario saber alguna de las tablas de multiplicar. La característica principal de este método es que se realiza un análisis de forma visual, de igual forma se pone en práctica la habilidad para realizar sumas (Porras \& Monge, 2012). A continuación, se describe el procedimiento:

Como primer paso, "se toma el primer número (multiplicando) y se ponen tantas rayas verticales como la cantidad de dígitos tenga el número" (Ver Figura 1).

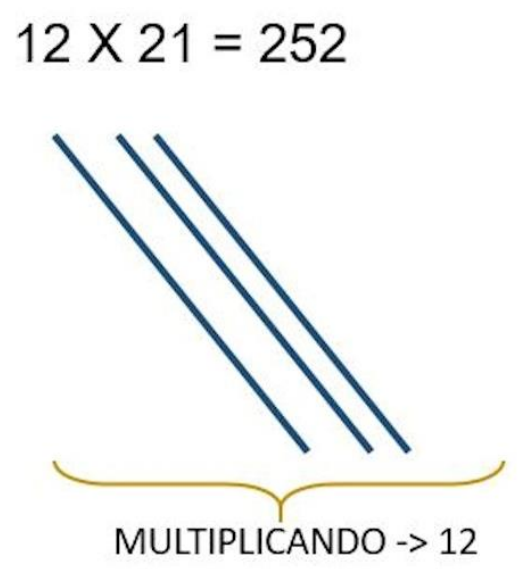

Figura 1 Representación del multiplicando, método maya Fuente: Elaboración propia (2019)
Para la parte del multiplicador "Se hace el mismo procedimiento del paso anterior, haciendo tantas rayas de forma vertical opuestas, a las del multiplicando según la cantidad de dígitos tenga el número" (Ver Figura 2).

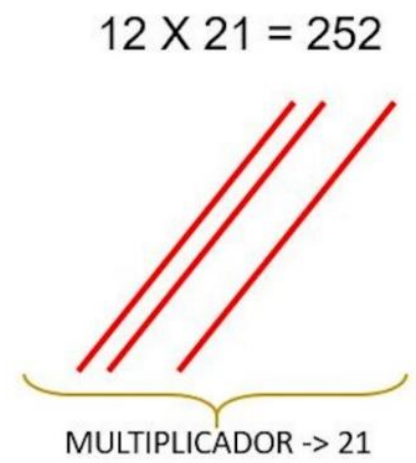

Figura 2 Representación del multiplicador, método maya Fuente: Elaboración Propia (2019)

Para obtener el resultado se marcan las intersecciones de las líneas creadas anteriormente, sumando cada una de las intersecciones de derecha a izquierda, en caso de que la suma sea mayor a 10, se anota el valor de la unidad en el valor de la decena, tal y como se realiza en la forma tradicional de multiplicar, por último, el resultado se anota de izquierda a derecha (Ver Figura 3).

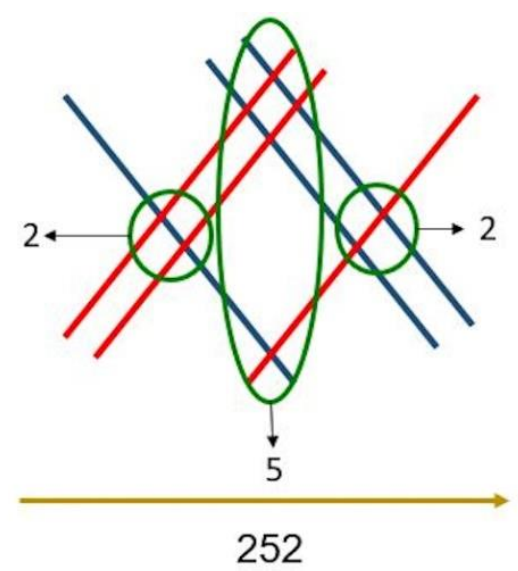

Figura 3 Representación del producto o resultado, método maya

Fuente: Elaboración Propia (2019)

\section{Diseño}

Para la elaboración de la aplicación incluyendo movimientos y diálogos que efectúa el robot NAO H25, se inició por el modelado de una secuencia didáctica (Ver Tabla 1), esta funciona como base para el desarrollo del contenido de la clase. 


\begin{tabular}{|c|c|}
\hline \multicolumn{2}{|c|}{ Secuencia Didáctica } \\
\hline Asignatura & $\begin{array}{l}\text { Matemáticas básicas } 3 \text { (educación } \\
\text { primaria). }\end{array}$ \\
\hline \multicolumn{2}{|c|}{$\begin{array}{l}\text { Implementación de una aplicación con el robot NAO H25 } \\
\text { para la enseñanza y comprobación de la operación } \\
\text { multiplicación. }\end{array}$} \\
\hline Finalidad & $\begin{array}{l}\text { Que el alumno aprenda a multiplicar y } \\
\text { realice la comprobación del resultado } \\
\text { por medio del método maya, todo por } \\
\text { medio de la clase impartida por el robo } \\
\text { NAO H25. }\end{array}$ \\
\hline Propósito & $\begin{array}{l}\text { Que el robot NAO H25 imparta una } \\
\text { clase a los alumnos, explicando una } \\
\text { forma de comprobar resultados } \\
\text { obtenidos de la multiplicación (método } \\
\text { maya), de tal forma que los alumnos } \\
\text { comprendan y puedan realizar un } \\
\text { ejercicio de dicho método. }\end{array}$ \\
\hline Duración & $\begin{array}{l}\text { Se realizará en una sola sesión, en un } \\
\text { tiempo máximo de } 1 \text { hora. }\end{array}$ \\
\hline Tema general & $\begin{array}{l}\text { Enseñanza de la operación } \\
\text { multiplicación y su comprobación por } \\
\text { el método maya. }\end{array}$ \\
\hline Contenidos & $\begin{array}{l}\text { Explicación por medio del robot NAO } \\
\text { H25 sobre una forma sencilla de } \\
\text { realizar la comprobación de resultados } \\
\text { de una multiplicación. }\end{array}$ \\
\hline \multicolumn{2}{|c|}{ Línea de la secuencia didáctica: } \\
\hline Apertura & $\begin{array}{l}\text { - Presentación del robot NAO } \\
\text { H25 y los motivos de la } \\
\text { sesión. }\end{array}$ \\
\hline Desarrollo & 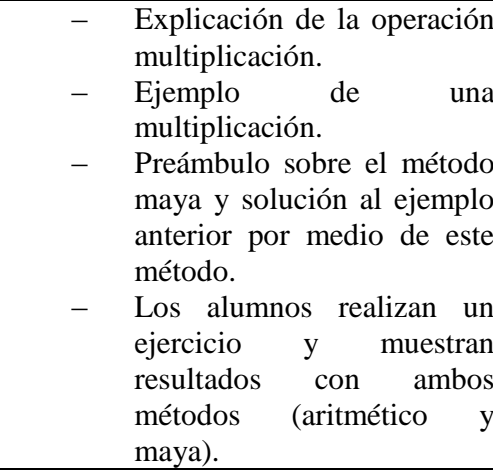 \\
\hline Cierre & $\begin{array}{l}\text { Resolver dudas a profesor y } \\
\text { alumnos sobre el tema } \\
\text { explicado. } \\
\text { Aplicación de encuestas a los } \\
\text { alumnos y evaluación de los } \\
\text { productos de aprendizaje. }\end{array}$ \\
\hline $\begin{array}{l}\text { Producto } \\
\text { aprendizaje }\end{array}$ & $\begin{array}{l}\text { La evaluación se realizará } \\
\text { por medio de un ejercicio } \\
\text { práctico junto con el llenado } \\
\text { de una encuesta donde } \\
\text { indiquen los alumnos que les } \\
\text { pareció la actividad. } \\
\end{array}$ \\
\hline
\end{tabular}

Tabla 1 Secuencia didáctica empleada para la clase Fuente: Elaboración Propia (2019)

Tomando como referencia el contenido de la secuencia didáctica, se realizó un diagrama de flujo que deje ver a detalle la programación textual y guiada de la aplicación, permitiendo observar que es lo que realizará el robot NAO H25 para impartir la clase (Ver Figura 4).

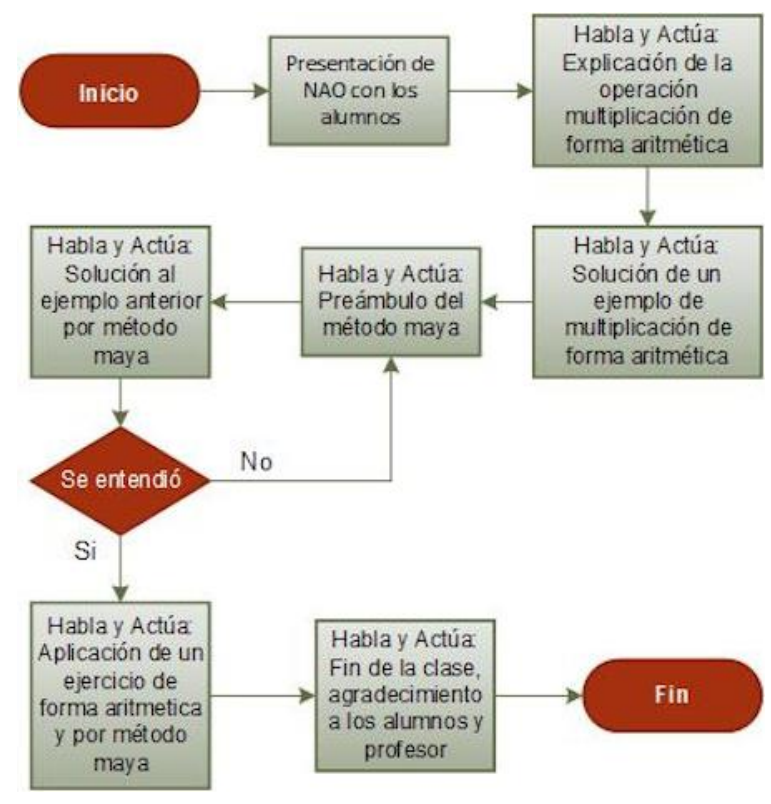

Figura 4 Diagrama de flujo de la programación de la aplicación

Fuente: Elaboración Propia (2019)

Con la finalidad de tener conocimiento acerca de la interacción de NAO con los alumnos y profesor, se formuló un Diagrama de Casos de Uso (Ver Figura 5), el cual muestra el comportamiento de NAO H25 con profesor y alumnos. Este diagrama se realizó con la aplicación ya montada en la memoria del robot NAO.

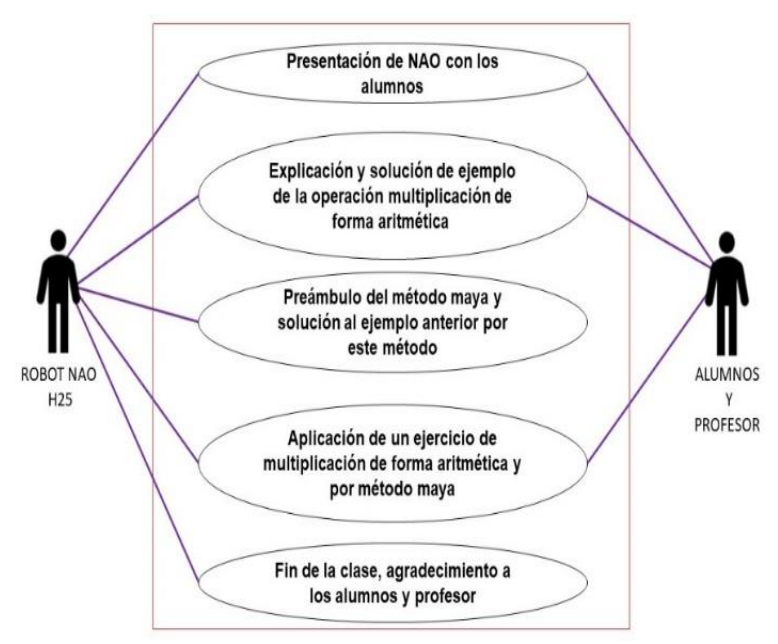

Figura 5 Diagrama de Casos de Uso

Fuente: Elaboración Propia (2019)

\section{Desarrollo}

Para la elaboración de la aplicación se utilizó el entorno de desarrollo Choregraphe (Ver Figura 6 y 7), este permite hacer una programación textual (por script) o una programación guida, con esta última podemos guardar los movimientos que deseamos que realice el robot NAO H25. 


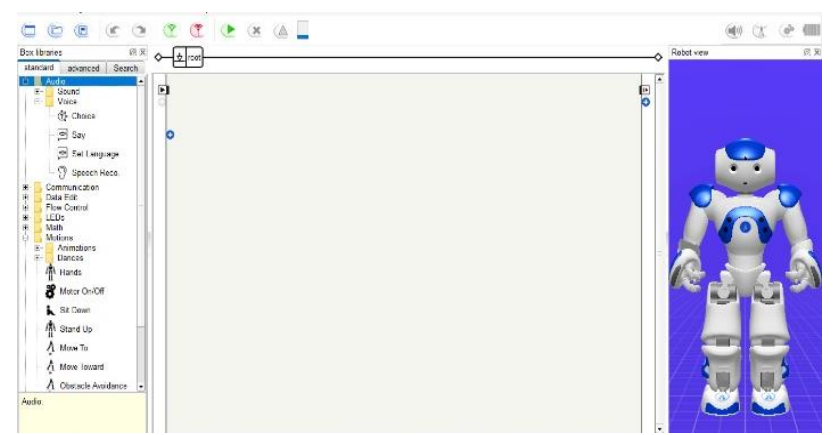

Figura 6 Entorno de desarrollo Choregraphe Fuente: Elaboración Propia (2019)

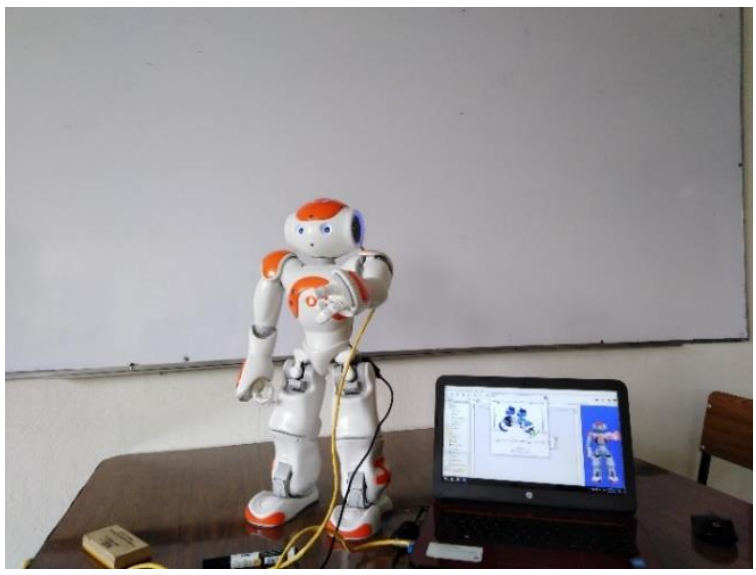

Figura 7 Ejemplo de programación guiada con Choregraphe

Fuente: Elaboración Propia (2019)

Como primer paso para el desarrollo de la aplicación, fue que NAO H25 sostuviera un marcador para pizarrón, este lo sostiene con sus dedos prensiles (Ver Figura 8 y 9).

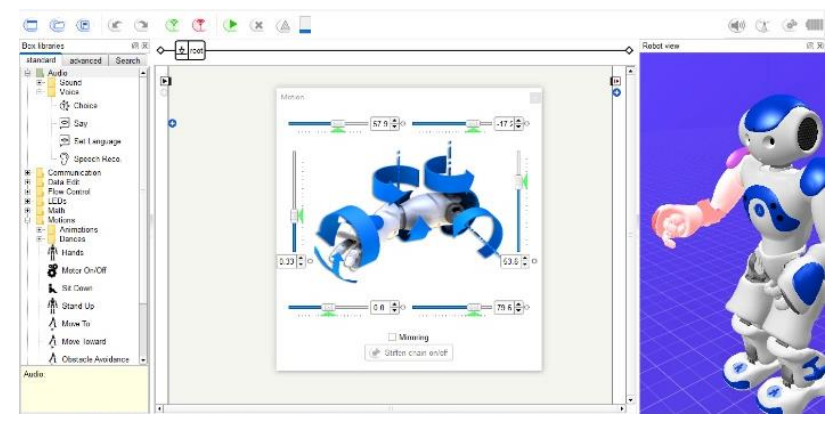

Figura 8 Forma de cómo se ajustó el brazo derecho de NAO H25 para sostener el marcador Fuente: Elaboración Propia (2019)

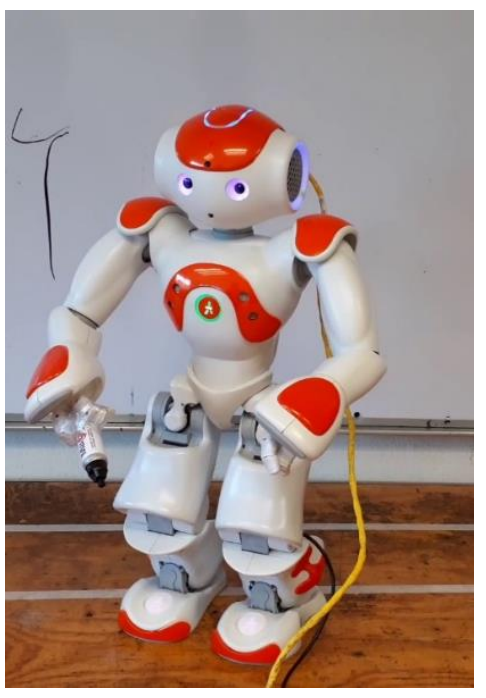

Figura 9 Muestra de NAO H25 sosteniendo un marcador Fuente: Elaboración propia (2019)

Una vez que NAO H25 sostiene el marcador, se trabajó en los movimientos que realizará al momento de explicar un ejemplo (Ver Figura 11). Toda la rutina que realiza NAO $\mathrm{H} 25$, va de acuerdo a la secuencia didáctica mencionada anteriormente. Cada uno de los módulos desarrollados en Choregraphe (Ver Figura 10) sirven para almacenar los movimientos que realiza $\mathrm{NAO}$, de igual forma hay módulos que funcionan para darle voz y pueda explicar el tema.

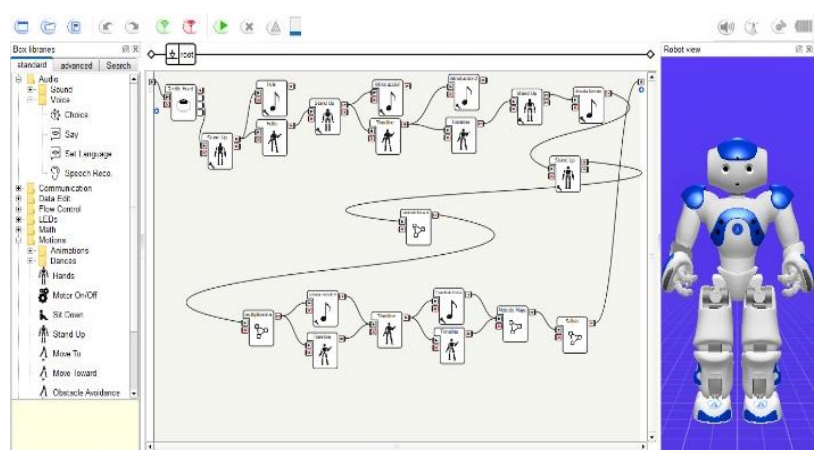

Figura 10 Módulos desarrollados en Choregraphe Fuente: Elaboración propia (2019)

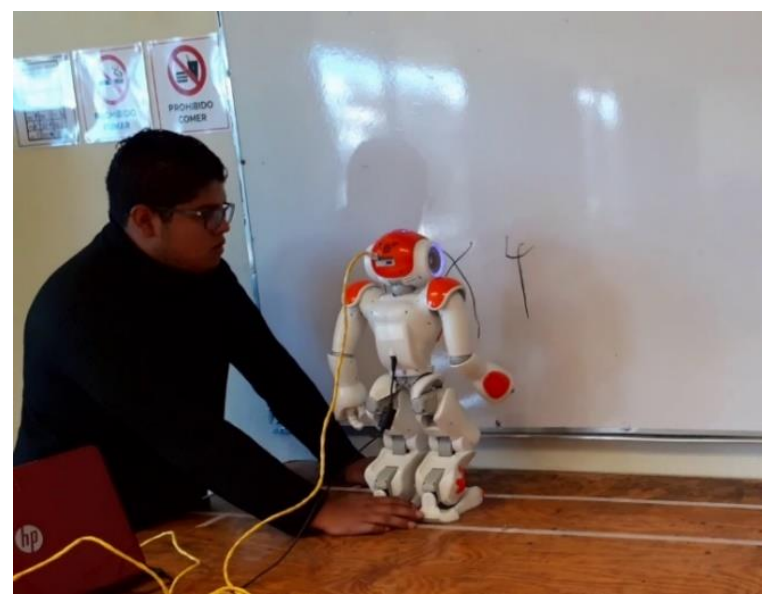

Figura 11Trabajo de movimientos Fuente: Elaboración Propia (2019)

GUTIERREZ-LUGO, Marco Antonio, MENDOZA-PÉREZ, Marco Alberto y CRUZ-FLORES, René Guadalupe. Desarrollo de una aplicación en el robot NAO H25 para la enseñanza y comprobación de la operación multiplicación con el método Maya. Revista de Aplicaciones de la Ingeniería. 2019. 
Para la elaboración del ejemplo de multiplicación aritmética se programaron distintos módulos (Ver Figura 12), cada uno de estos realiza un movimiento como sostener el marcador, escribir en el pizarrón o hablar para presentarse ante el público. Al unir y ejecutar todos los módulos, podemos observar cómo queda el ejemplo de la multiplicación (Ver Figura 13).



Figura 12 Módulos realizados para el ejemplo de la multiplicación aritmética

Fuente: Elaboración propia (2019)



Figura 13 Ejemplo de multiplicación aritmética, realizado por NAO H25

Fuente: Elaboración Propia (2019)

Continuando con el desarrollo de la aplicación, se prosiguió con la realización de la comprobación maya (Ver Figura 14), esta parte, mencionada en la secuencia didáctica se llevará acabó con el método maya, con lo cual el robot NAO H25 tendrá que dibujar líneas verticales para el multiplicador y líneas horizontales para el multiplicando. En la Figura 15, se muestra el ejemplo de la comprobación con el método maya, el robot escribe el procedimiento y el resultado. En la Figura 16, se muestra la aplicación terminada.

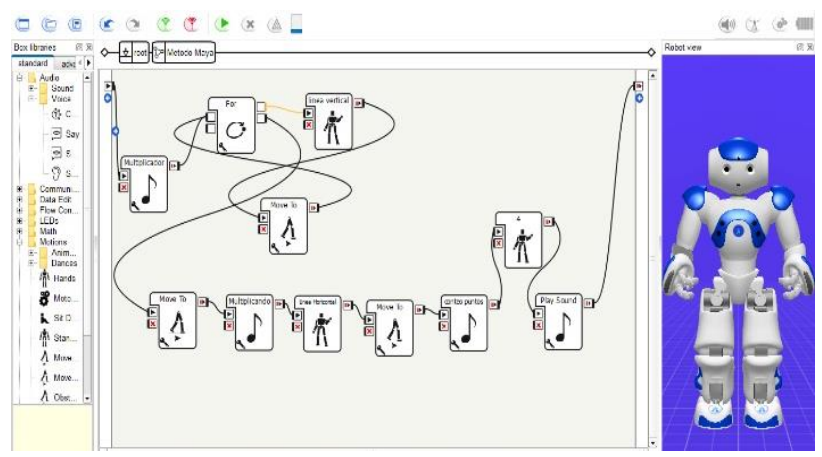

Figura 14 Módulos programados que ejecutan el método maya

Fuente: Elaboración propia (2019)

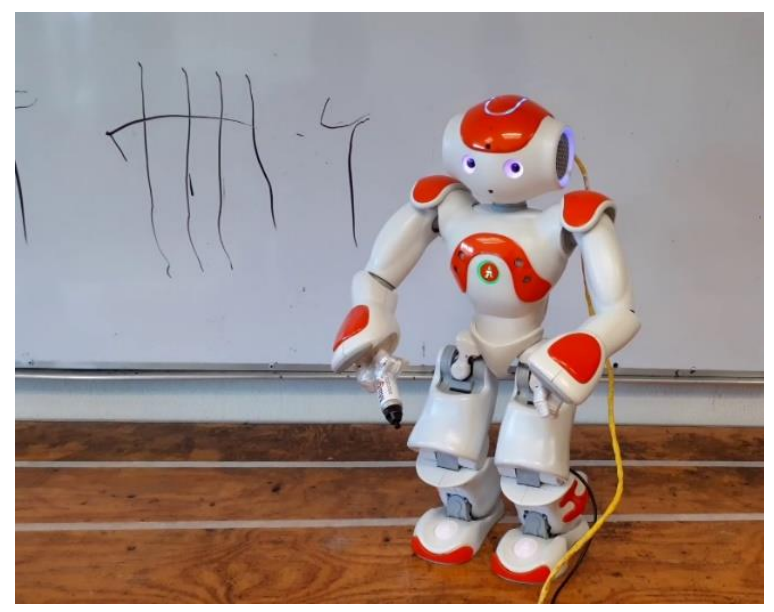

Figura 15 Método maya realizado por el robot NAO H25 Fuente: Elaboración Propia (2019)

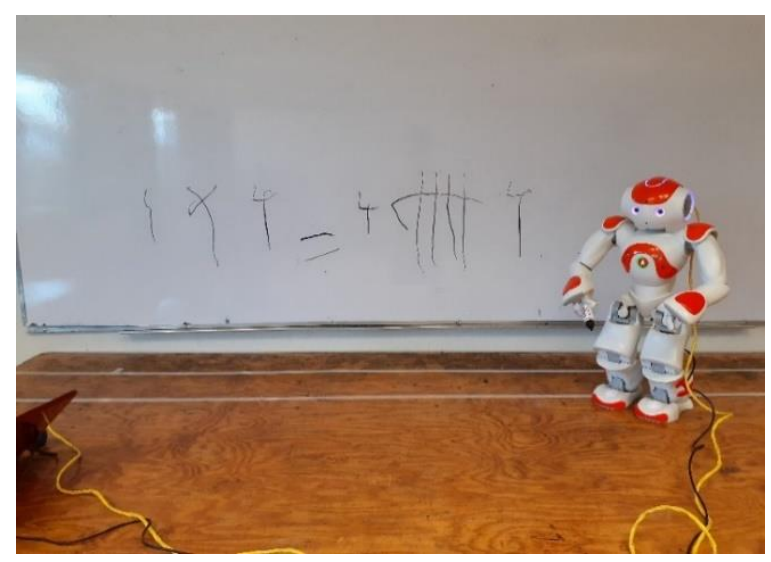

Figura 16 Ejemplo de la aplicación terminada Fuente: Elaboración propia (2019)

\section{Resultados}

\section{Implementación}

Se realizaron pruebas en un grupo de 32 alumnos de nuevo ingreso y un maestro de la carrera de Ingeniería en Computación del Centro Universitario UAEM Valle de Chalco. En la implementación se mostró la rutina completa que realiza el robot $\mathrm{NAO} \mathrm{H} 25$, en donde explica la multiplicación aritmética, una forma de comprobación por el método maya y la solución del ejercicio (Ver Figura 17 y 18).

GUTIERREZ-LUGO, Marco Antonio, MENDOZA-PÉREZ, Marco Alberto y CRUZ-FLORES, René Guadalupe. Desarrollo de una aplicación en el robot NAO H25 para la enseñanza y comprobación de la operación multiplicación con el método Maya. Revista de Aplicaciones de la Ingeniería. 2019 


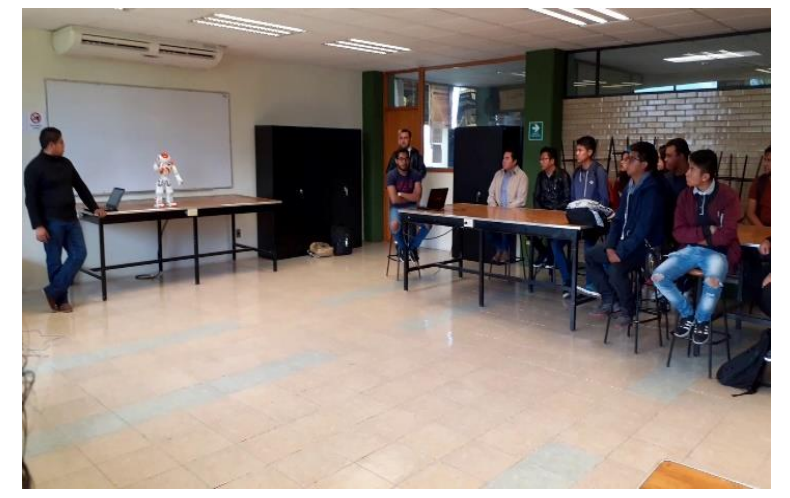

Figura 17 Breve introducción sobre la problemática y la aplicación

Fuente: Elaboración propia (2019)

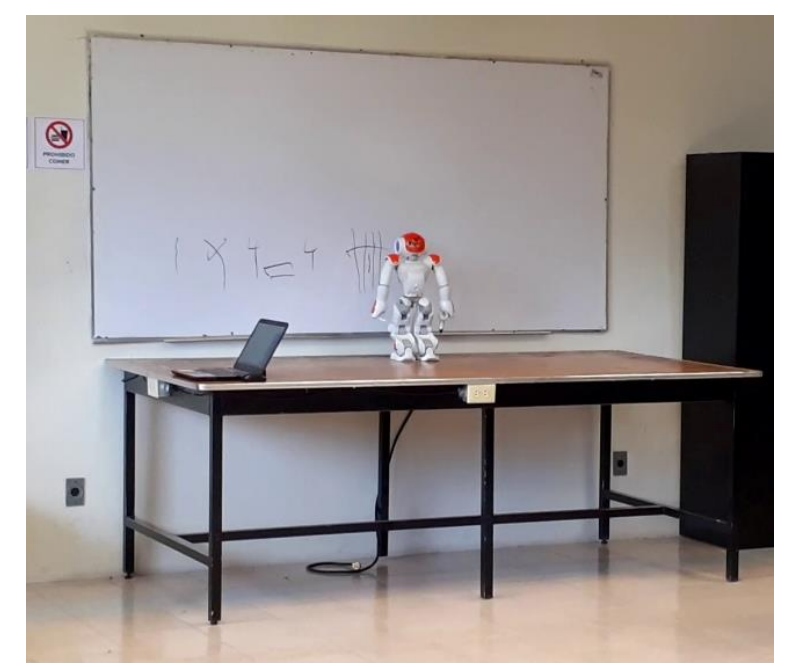

Figura 18. Ejecución de la aplicación.

Fuente: Elaboración propia (2019)

\section{Evaluación}

La evaluación se llevó acabo por medio de una encuesta (Ver Figura 19) en donde se aplicaron seis preguntas, para saber si la aplicación es amigable, innovadora, funcional y entendible.



Figura 19 Muestra de la encuesta que se realizó a los alumnos

Fuente: Elaboración Propia (2019)
Una vez terminada la encuesta se obtuvieron los siguientes resultados: La primera pregunta de la encuesta menciona si es fácil de comprender la multiplicación aritmética con el robot NAO, se obtuvo una respuesta favorable, con un $75 \%$ de los alumnos que quedaron satisfechos con la ejecución de la aplicación, el otro $25 \%$ no se convencieron del todo (Ver Gráfico 1).

\section{1.- Fue fácil comprender la multiplicación con el robot NAO.}

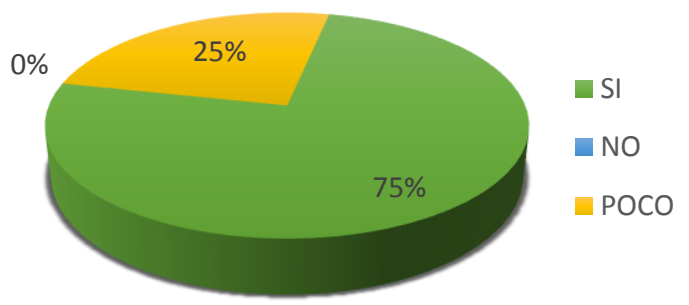

Gráfico 1 Resultados de la pregunta 1

Fuente: Elaboración propia (2019)

El método maya fue explicado por NAO $\mathrm{H} 25$, mencionado como se usa este método y que partes lo conforman, para un $88 \%$ de los alumnos encuestados fue sencillo el comprender el método, mientras que un $9 \%$ se quedó con alguna duda de cómo aplicar el método (Ver Gráfico 2).

\section{2.- El método maya fue sencillo de comprender con el robot NAO.}



Gráfico 2 Resultados de la pregunta 2 Fuente: Elaboración Propia (2019)

A la mayor parte de los alumnos encuestados (94\% de los alumnos) se le hizo interesante que el robot NAO explicara el tema (Ver Gráfico 3), de igual forma se obtuvieron resultados favorables en la implementación que se mostró con el robot NAO H25 (Ver Gráfico $4)$. 
3.- Se te hizo interesante que este tema fue explicacdo por el robot NAO.

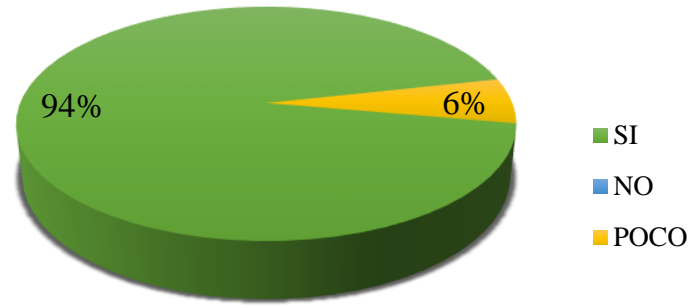

Gráfico 3. Resultados a la pregunta 3

Fuente: Elaboración propia (2019)

4.- Te gusto como se implementó la aplicación en el robot NAO.

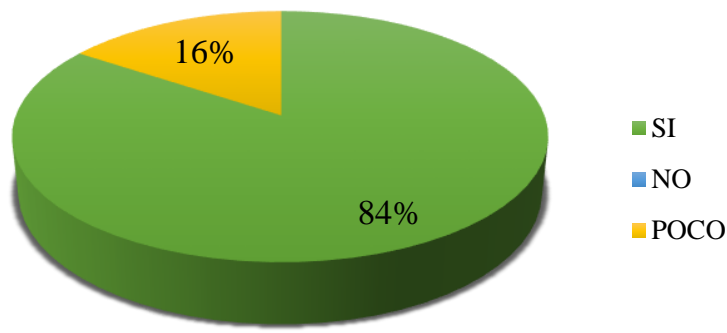

Gráfico 4. Resultados a la pregunta 4

Fuente: Elaboración propia (2019)

Un $97 \%$ de los alumnos encuestados quieren que se desarrollen más aplicaciones como esta, para fomentar el uso de las tecnologías y un mejor aprendizaje en todos los niveles de educación (Ver Gráfico 5).

\section{5.- Te gustaría que el robot NAO explicara más temas.}

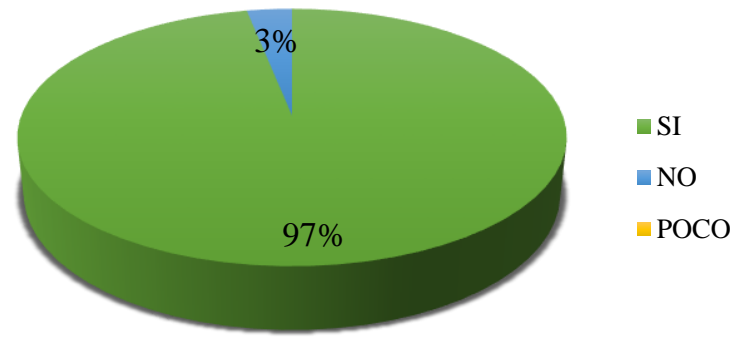

Gráfico 5. Resultados a la pregunta 5 Fuente: Elaboración propia (2019).

Un $84 \%$ de los alumnos creen que el implementar la aplicación en estudiantes de primaria es de gran utilidad para aprender y comprender mejor la multiplicación (Ver Gráfico 6).

\section{6.- Crees que la aplicación sería de gran utilidad para un mejor aprendizaje de la multiplicación.}

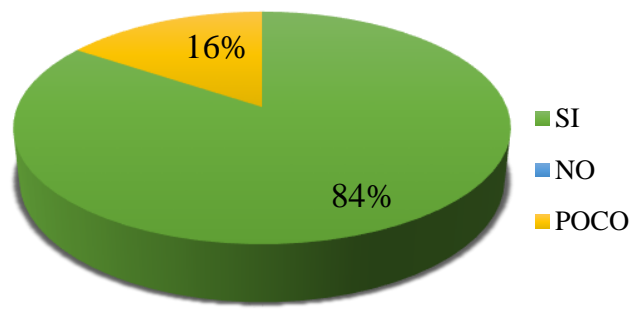

Gráfico 6. Resultados a la pregunta 6

Fuente: Elaboración Propia (2019)

La aplicación cuenta con una buena aceptación, debido a la forma en como se explica el tema de las multiplicaciones, siendo una manera novedosa de impartir un tema, sin en cambio se tiene que mejorar los trazos o dibujos que realiza el robot NAO H25 (puede ser el tiempo y fuerza de los motores del brazo que tiene el marcador), debido a la distancia que hay entre el brazo que sostiene el marcador con respecto al pizarrón.

\section{Conclusiones}

La presentación realizada por el robot NAO H25 al grupo de 32 alumnos de nuevo ingreso y un maestro de la carrera de Ingeniería en Computación del Centro Universitario UAEM Valle de Chalco, mostro la ejecución de la aplicación desarrollada, donde al ver los resultados de la encuesta aplicada, se llegó a la conclusión de que la explicación realizada por el robot NAO H25 es novedosa y funcional, esta explicación se apega a la secuencia didáctica elaborada para la clase impartida por el robot NAO H25.

Otro de los puntos que se obtuvieron fue que la aplicación desarrollada es de gran utilidad y que sirve para un mejor aprendizaje de la operación aritmética multiplicación, teniendo en cuenta lo anterior podemos concluir que la aplicación es creativa, de fácil comprensión y de gran interés por los alumnos de educación básica, gracias a que con ayuda de NAO H25 la aplicación es más vistosa, esto da hincapié a que NAO H25 explique más temas de la misma disciplina o de diferentes. 
A lo largo de la carrera de Ingeniería en Computación se cursan Unidades de Aprendizaje de la línea de acentuación de Interacción Hombre - Máquina, las cuales proporcionan conocimientos declarativos y procedimentales, desde la identificación de los componentes de un robot, su ensamble, conexión, configuración, y programación textual y guiada de cualquier tipo de robot.

Por último, para los alumnos de nuevo ingreso, al no tener ningún contacto anterior con $\mathrm{NAO}$ o algún robot del tipo humanoide quedaron complacidos con la aplicación, de igual forma se mostró que NAO es capaz de realizar distintas actividades, que estén relacionadas con la educación o no. El desarrollo de esta aplicación queda como una puerta abierta para que nuevos estudiantes se animen a desarrollar e innovar nuevas aplicaciones para el robot NAO H25.

\section{Agradecimiento}

A la Universidad Autónoma del Estado de México.

\section{Referencias Bibliográficas}

Anonimo. (s.f.). Concepción de Enseñanza / Aprendizaje. Obtenido de http://www.unter.org.ar/imagenes/10061.pdf

Arias, A. (1999). Las Estrategias de Aprendizaje. Revision Teorica y Conceptual. Revista Latinoamericana de Psicología, 425461.

BeJob Santillana. (2017). ¿Qué es la programación robótica y para qué sirve? Obtenido de https://www.bejob.com/que-es-laprogramacion-robotica-y-para-que-sirve- $2 /$

Capek, K. (2004). R.U.R. Robots Universales Rossum. Barcelona: Círculo de Lectores.

Centeno, P. (2017). Una experiencia de estandarización utilizando el modelo ADDIE en la elaboración de guías temáticas. Costa Rica: eCiencias de la Información.

Esquivel, I. (2014). Los Modelos TecnoEducativos, revolucionando el aprendizaje del siglo XXI. México.

García, E. (2009). Aprendizaje y construcción del conocimiento, Madrid: Las plataformas de aprendizaje.
Juárez, C., Sánchez, J., \& Mendoza, M. (2017). Manual para prácticas de laboratorio, manejo del robot NAO H25. México.

Mediatec, G. (2012). NAO H25. Obtenido de http://www.grupo-

mediatec.com/robotica/h25.html

Palma, I., Buades, J., \& Guerrero, A. (2019). Interaccion basada en robots sociales para la evaluacion de expresiones faciales.

Pinto, M., Barrera, N., \& Pérez, W. (2010). Uso de la Robótica Educativa como Herramienta en los Procesos de Enseñanza. Colombia.

Porrras, A., \& Monge, C. (2012). Un viaje por los diversos métodos de multiplicar . Costa Rica: VIII FESTIVAL INTERNACIONAL DE MATEMÁTICA .

Reyes, F. (2011). Robótica. Control de robots manipuladores. México: Alfaomega.

Rodríguez, A. (2011). Programación de Robot. Obtenido de Automática Industrial: https://automaticaindustrial.wordpress.com/rob otica/programacion-de-robot/

Santiago, R. (2015). the flipped classroom. Obtenido de https://www.theflippedclassroom.es/uninfografico-sobre-el-modelo-addie/

Secretaria de Educación Pública. (2004). Desafíos matemáticos. Libro para el maestro. Ciudad de México: Secretaria de Educación Pública.

Solís, J. (2001). SemanticWebBuilder. Obtenido de

http://www.semanticwebbuilder.org.mx/es_mx/ swb/Sistemas_Embebidos_Innovando_hacia_lo s_Sistemas_Inteligentes_

Valencia, U. I. (2018). El aprendizaje situado: un enfoque social y orientado al contexto. Obtenido de https://www.universidadviu.com/elaprendizaje-situado-un-enfoque-social-yorientado-al-contexto/

Velasco, M. (2010). Estrategias didácticas para el Aprendizaje Colaborativo. 